\title{
INTERVALO HÍDRICO ÓTIMO DE UM LATOSSOLO VERMELHO DISTRÓFICO, APÓS O PRIMEIRO PERÍODO DE PASTEJO CONTÍNUO DE Brachiaria ruziziensis, EM SISTEMA INTEGRAÇÃO LAVOURA-PECUÁRIA ${ }^{(1)}$
}

\author{
Jonez Fidalski ${ }^{(2)}$, Cássio Antonio Tormena ${ }^{(3)}$ \& Sérgio José Alves ${ }^{(4)}$
}

\begin{abstract}
RESUMO
O intervalo hídrico ótimo (IHO) destaca-se como um dos melhores indicadores da qualidade física do solo, sob sistemas intensivos de produção. $O$ objetivo deste trabalho foi avaliar a qualidade física de um Latossolo Vermelho distrófico típico por meio do IHO, após três safras agrícolas de lavoura e o primeiro ano de sistema integração lavoura-pecuária, em Xambrê, noroeste do Paraná. O experimento foi implantado em blocos casualizados, com três repetições. Os tratamentos consistiram de quatro alturas de pastejo $(0,10 ; 0,20 ; 0,30 ; \mathrm{e} 0,40 \mathrm{~m})$ e um tratamento testemunha sem pastejo. A braquiária (Brachiaria ruziziensis) foi semeada em março de 2010 e o pastejo contínuo de bovinos foi realizado durante 110 dias (março-setembro). Em outubro desse ano, coletaram-se 90 amostras de solo com cilindros metálicos $(0,05 \mathrm{~m}$ de altura e 0,05 m de diâmetro) no centro das camadas de 0-0,10; 0,10-0,20; e 0,20-0,30 m. Essas amostras foram utilizadas para obter a curva de retenção de água, a curva de resistência do solo à penetração e a densidade do solo (Ds); a partir dessas, foi calculado o IHO e obtida a densidade crítica do solo (Dsc). Utilizaram-se os limites críticos de $-80 \mathrm{hPa}$, para a capacidade de campo $\left(\theta_{\mathrm{cc}}\right) ;-15.000 \mathrm{hPa}$, para o ponto de murcha permanente $\left(\theta_{\mathrm{pmp}}\right) ; 2,5 \mathrm{MPa}$, para a resistência do solo à penetração $\left(\theta_{\mathrm{rp}}\right)$; $\mathrm{e}$ $0,10 \mathrm{~m}^{3} \mathrm{~m}^{-3}$, para a porosidade de aeração $\left(\theta_{\mathrm{pa}}\right)$. O IHO foi maior a 0-0,10 m e a RP foi o fator de maior limitação do IHO em todas as camadas, especialmente a 0,10-0,20 e 0,20-0,30 m. As Dsc decresceram em profundidade de 1,66; 1,64; e $1,62 \mathrm{Mg} \mathrm{m}^{-3}$, respectivamente, para as camadas de $0-0,10 ; 0,10-0,20 ;$ e $0,20-0,30 \mathrm{~m}$. O manejo desse solo sob sistema integração lavoura-pecuária com altura de pastejo de $0,10 \mathrm{~m}$ apresentou a maior frequência de amostras de solo com Ds $\geq$ Dsc.
\end{abstract}

Termos de indexação: água no solo, arenito Caiuá, curva de retenção de água, densidade do solo, manejo do solo, qualidade do solo, resistência do solo à penetração.

\footnotetext{
(1) Recebido para publicação em 24 de julho de 2012 e aprovado em 20 de março de 2013.

(2) Pesquisador, Instituto Agronômico do Paraná - IAPAR. Bolsista da Fundação Araucária. Caixa Postal 564. CEP 87701-970 Paranavaí (PR). E-mail: fidalski@iapar.br

(3) Professor Associado do Departamento de Agronomia, Universidade Estadual de Maringá - UEM. Bolsista do CNPq. Av. Colombo, 5790. CEP 87020-900 Maringá (PR). E-mail: catormena@uem.br

(4) Pesquisador, IAPAR. Bolsista da Fundação Araucária. Caixa Postal 481. CEP 86001-970 Londrina (PR). E-mail: sja@iapar.br
} 


\title{
SUMMARY: LEAST LIMITING WATER RANGE IN A TYPIC HAPLORTHOX AFTER THE FIRST CONTINUOUS GRAZING PERIOD OF Brachiaria ruziziensis IN INTEGRATED CROP-LIVESTOCKSYSTEM
}

\begin{abstract}
The least limiting water range (LLWR) is one of the best indicators of soil physical quality in intensive production systems. The objective of this study was to evaluate the physical quality of a Typic Haplorthox by the LLWR after three crop growing seasons and the first year of crop-livestock integration in Xambrê, northwestern Paraná, Brazil. The experiment was established in a randomized block design with three replications. Treatments consisted of four brachiaria heights before grazing $(0.10 ; 0.20 ; 0.30$, and $0.40 \mathrm{~m}$ ) and a control treatment without grazing. The brachiaria (Brachiaria ruziziensis) was sown in March 2010 and cattle grazed on it continuously for 110 days (March-September). In October 2010, 90 soil samples were collected with metal cylinders (height $0.05 \mathrm{~m}$, diameter $0.05 \mathrm{~m}$ ) from the center of the layers 0-0.10;0.10-0.20;0.20-0.30 m. These samples were used to obtain the water retention curve, the penetration resistance curve and the soil bulk density $\left(B_{d}\right)$, from which the LLWR and the critical bulk density $\left(B_{d c}\right)$ were calculated. The critical limits of $-80 h P a$ for field capacity $\left(\theta_{f c}\right),-15,000 \mathrm{hPa}$ for wilting point $\left(\theta_{w p}\right), 2.5 \mathrm{MPa}$ for soil resistance to penetration $\left(\theta_{s r}\right)$ and $0.10 \mathrm{~m}^{3} \mathrm{~m}^{-3}$ for air filled porosity $\left(\theta_{\text {afp }}\right)$ were used. The LLWR was highest in 0-0.10 $\mathrm{m}$ and $\theta_{\text {sr }}$ was the most relevant variable to reduce LLWR in all layers, especially the layers 0.10-0.20 $\mathrm{m}$ and 0.20-0.30 $\mathrm{m}$. The $B d_{c}$ decreased with depth for values of $1.68,1.65$ and $1.64 \mathrm{Mg} \mathrm{m}^{-3}$, respectively, in the layers $0-0.10,0.10-0.20$ and $0.20-0.30 \mathrm{~m}$. The management of this soil under integrated crop livestock at a grazing height of $0.10 \mathrm{~m}$ had the highest frequency of soil samples with $B_{d} \geq B_{d c}$.

Index terms: soil water, Caiuá Sandstone, soil bulk density, water retention curve, soil management, soil quality, resistance to penetration.
\end{abstract}

\section{INTRODUÇÃO}

A qualidade física do solo pode ser comprometida em sistemas de integração lavoura-pecuária (Petean et al., 2010; Collares et al., 2011) ou ser mantida (Marchão et al., 2007; Spera et al., 2010; Conte et al., 2011), em razão do manejo das forrageiras e dos animais nesse sistema de produção. Um dos princípios básicos no estudo do impacto de sistemas de manejo na qualidade do solo consiste em avaliá-la especificamente para cada classe e sistema manejo de solo (Karlen et al., 1997; Sojka \& Upchurch, 1999).

Vários estudos consideraram a densidade (Ds) e a porosidade e água $(\theta)$ do solo para avaliar sistemas de integração lavoura-pecuária (Marchão et al., 2007; Collares et al., 2011). Com os avanços na conceituação de qualidade física do solo, surgiram vários indicadores, destacando-se o intervalo hídrico ótimo (IHO), que vem sendo utilizado em sistema integração lavoura-pecuária (Serafim et al., 2008; Petean et al., 2010; Calonego et al., 2011). De acordo com Tormena et al. (2007), o IHO é determinado a partir das curvas de retenção de água do solo e da curva de resistência do solo à penetração, para cada valor de Ds, na capacidade de campo $\left(\theta_{\mathrm{cc}}\right)$, no ponto de murcha permanente $\left(\theta_{\text {pmp }}\right)$, para determinado valor de resistência do solo à penetração $\left(\theta_{\mathrm{rp}}\right)$ e porosidade livre de água mínima estimada para manter adequada aeração no solo $\left(\theta_{\mathrm{pa}}\right)$.

Os solos originários de arenito, no noroeste do Paraná, têm área de 3,2 milhões de hectares e representam $16 \%$ da área do Estado (Sá \& Caviglione, 1999), dos quais aproximadamente $70 \%$ são ocupados por pastagens. Um estudo conduzido por Fidalski et al. (2008a) demonstrou que o pastejo em sistema de lotação contínua com controle da taxa de lotação animal, em razão da produção de forragem de capim coast cross (Cynodon dactylon), consorciada ou não com leguminosa amendoim forrageiro (Arachis pintoi), não comprometeu a qualidade física de um Latossolo Vermelho distrófico. Sarmento et al. (2008), em um solo similar com pastagem Panicum maximum, sob diferentes taxas de lotação animal, em razão de doses crescentes de nitrogênio, obtiveram por meio de análise de regressão, na camada de $0-0,10 \mathrm{~m}$, a DS máxima de $1,64 \mathrm{Mg} \mathrm{m}^{-3}$. Em Latossolo Vermelho distrófico textura média, Cavalieri et al. (2006) obtiveram densidade crítica do solo (Dsc) de 1,65 e $1,62 \mathrm{Mg} \mathrm{m}^{-3}$, respectivamente, para as camadas de 0-15 e 0,15-0,30 m; a Dsc corresponde a Ds em que o IHO é nulo (Tormena et al., 1998, 1999).

O objetivo deste trabalho foi avaliar a qualidade física, por meio do IHO, de um Latossolo Vermelho distrófico típico, após três safras agrícolas de lavoura e o primeiro ano de sistema integração lavourapecuária no noroeste do Paraná.

\section{MATERIAL E MÉTODOS}

O trabalho foi realizado em um experimento de 14 ha de integração lavoura-pecuária, na Estação 
Experimental do Instituto Agronômico do Paraná (IAPAR), no município de Xambrê ( $23^{\circ} 44^{\prime}$ 'S e $53^{\circ} 29^{\prime}$ W), no noroeste do Paraná. O clima é classificado como subtropical (Cfa), de acordo com Köppen (Caviglione et al., 2000). O solo é um Latossolo Vermelho distrófico típico (Santos et al., 2006), textura arenosa/média, característico da região noroeste do Paraná (Embrapa, 1984; Fasolo et al., 1998), em relevo suave-ondulado, cujas características estão descritas no quadro 1.

O sistema de integração lavoura-pecuária foi implantado na safra agrícola de 2009/2010 com a cultura da soja no verão e braquiária (Brachiaria ruziziensis) no inverno, após três safras agrícolas de soja no verão e aveia no inverso sem pastejo (2006/ 2007 a 2008/2009).

O experimento constituído de cinco tratamentos foi instalado em delineamento de blocos casualizados com três repetições. Cada parcela experimental tinha aproximadamente área de 1 ha. Os tratamentos apresentavam quatro alturas de pastejo de $B$. ruziziensis $(0,10 ; 0,20 ; 0,30 ; \mathrm{e} 0,40 \mathrm{~m})$ e um tratamento testemunha sem pastejo.

A B. ruziziensis foi semeada em 06/03/2010 e o pastejo contínuo de bovinos foi realizado durante 14/ 05/2010 a 01/09/2010, totalizando 110 dias de pastejo. A entrada dos animais para pastejo foi feita quando a forrageira atingiu, em média, $0,30 \mathrm{~m}$ de altura (cerca de $3.000 \mathrm{~kg} \mathrm{ha}^{-1}$ de matéria seca). A manutenção das alturas propostas foi realizada pela variação na quantidade de animais em pastejo. $\mathrm{O}$ método de pastejo utilizado foi lotação contínua, com carga animal variável, por meio da utilização da técnica put and take, descrita por Mott \& Lucas (1952), mantendo-se fixa a quantidade de dois animais experimentais por parcela e o número variável de animais reguladores. $\mathrm{O}$ ajuste da carga animal foi feito semanalmente com a entrada ou retirada de animais reguladores para manter a altura da pastagem o mais próximo da pretendida (Aguinaga et al., 2006). A caracterização média da lotação e disponibilidade de forragem por tratamento de altura de pastejo durante o primeiro período de pastejo estão caracterizadas no quadro 2 .

A amostragem de solo foi realizada em outubro de 2010. As amostras indeformadas de solo foram coletadas com cilindros metálicos $(0,05 \mathrm{~m}$ de altura e $0,05 \mathrm{~m}$ de diâmetro) no centro das camadas de $0-0,10$; 0,10-0,20; e 0,20-0,30 m, em duplicata, totalizando 90 amostras de solo. Um conjunto de 45 amostras de solo indeformadas de cada tratamento e camada de solo foi saturado e submetido aos potenciais $(\psi)$ de -10 , $-20,-40,-60,-80$ e $-100 \mathrm{hPa}$ em mesa de tensão (Claessen, 1997) e por secamento natural em ambiente controlado, conseguindo-se $\psi$ entre -282 e - $816 \mathrm{hPa}$, obtidos com tensiômetro em miniatura modelo T5 (Eijkelkamp, 2012) e $\psi$ entre -2300 e $31200 \mathrm{hPa}$, medidos com psicrômetro modelo WP4-T (Klein et al., 2010). Em seguida, as amostras foram secadas em estufa a $105^{\circ} \mathrm{C}$ durante $48 \mathrm{~h}$ para obter a massa de água e a massa de solo. A Ds foi obtida pela razão entre a massa de sólidos e o volume dos cilindros metálicos. As curvas de retenção de água do solo foram ajustadas pelo modelo matemático proposto por van Genuchten (1980) com a restrição $(m=1-1 / n): \theta=\theta \mathrm{r}+\left\{(\theta \mathrm{s}-\theta \mathrm{r}) /\left[1+(\alpha \psi)^{n}\right]^{(1-1 / n)}\right\}$, em que $\psi(\mathrm{hPa})$, $\theta, \theta \mathrm{r}$ e $\theta \mathrm{s}\left(\mathrm{m}^{3} \mathrm{~m}^{-3}\right)$ e a $\left(\mathrm{hPa}^{-1}\right)$ e $n$ são coeficientes do modelo. A Ds foi incorporada nas curvas de retenção de água do solo, conforme Tormena \& Silva (2002). Os coeficientes do modelo van Genuchten $(\theta, n, \theta$ s e $\theta$ r) foram obtidos com a rotina PROC NLIN (SAS, 1999).

Um segundo conjunto de 45 amostras indeformadas de solo coletado em duplicata nos cinco tratamentos, nos três blocos e nas três profundidades foi saturado; as amostras foram secas ao ar para obter ampla variabilidade da umidade do solo e de resistência do solo à penetração (RP), as quais foram feitas por meio de um minipenetrômetro dinâmico, similar ao desenvolvido por Sá et al. (2007), seguido do secamento dessas em estufa a $105^{\circ} \mathrm{C}$, durante $48 \mathrm{~h}$, para obter a Ds e o $\theta$. A relação funcional entre RP, $\theta$ e Ds foi ajustada utilizando a função proposta por Busscher (1990), a partir dos procedimentos descritos por Silva et al. (1994): $\mathrm{RP}=e \theta^{f} \mathrm{Ds}^{g}$, cuja solução foi obtida por meio da transformação logarítmica em uma equação linear múltipla: $\ln (\mathrm{RP})=\ln (e)+f(\ln \theta)+g(\ln D s)$, em que: $\mathrm{RP}(\mathrm{MPa}), \theta\left(\mathrm{m}^{3} \mathrm{~m}^{-3}\right)$ e $\mathrm{Ds}\left(\mathrm{Mg} \mathrm{m}^{-3}\right)$ e os coeficientes de ajuste dos dados ao modelo matemático são $e, f$ e $g$. A equação foi linearizada e os ajustes dos modelos das curvas de resistência do solo à penetração foram feitos com a rotina PROC REG (SAS, 1999).

Quadro 1. Características do Latossolo Vermelho distrófico típico em sistema de integração lavoura-pecuária

\begin{tabular}{|c|c|c|c|c|c|c|c|c|c|}
\hline \multirow{2}{*}{ Camada } & \multirow{2}{*}{ Horizonte } & \multirow{2}{*}{ Argila } & \multirow{2}{*}{ Silte } & \multicolumn{3}{|c|}{ Areia } & \multirow{2}{*}{ Classe textural $^{(1)}$} & \multirow{2}{*}{$\mathbf{V}$} & \multirow{2}{*}{ Carbono orgânico } \\
\hline & & & & Total & Fina & Grossa & & & \\
\hline $\mathrm{m}$ & & & 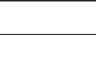 & $\mathrm{g} \mathrm{kg}^{-1}$ & & & & $\%$ & $\mathrm{~g} \mathrm{~kg}^{-1}$ \\
\hline $0-0,10$ & $\mathrm{~A}$ & 100 & 30 & 870 & 350 & 650 & Arenosa & 46 & 7,05 \\
\hline $0,11-0,20$ & $\mathrm{AB}$ & 120 & 20 & 860 & 350 & 650 & Arenosa & 42 & 5,98 \\
\hline $0,21-0,50$ & Bw1 & 160 & 10 & 830 & 340 & 660 & Média & 26 & 4,10 \\
\hline $0,51-2,00$ & Bw2 & 170 & 10 & 820 & 360 & 640 & Média & 9 & 2,83 \\
\hline
\end{tabular}

(1) Santos et al. (2006). 
Quadro 2. Caracterização média da lotação, número de bovinos e disponibilidade de forragem por tratamento de altura de pastejo durante o primeiro período de pastejo de 110 dias (14/05/2010 a 01/09/2010)

\begin{tabular}{lcccc}
\hline \multirow{2}{*}{ Descrição } & \multicolumn{3}{c}{ Altura de pastejo (m) } \\
\cline { 2 - 5 } & $\mathbf{0 , 1 0}$ & $\mathbf{0 , 2 0}$ & $\mathbf{0 , 3 0}$ & $\mathbf{0 , 4 0}$ \\
\hline Lotação $\left(\mathrm{UA} \mathrm{ha}^{-1}\right)^{(1)}$ & 2,6 & 2,4 & 1,9 & 1,7 \\
Número de bovinos (unidade ha $\left.{ }^{-1}\right)$ & 5,7 & 5,3 & 4,0 & 4,0 \\
Disponibilidade de forragem $\left(\mathrm{Mg} \mathrm{ha}^{-1}\right)$ & 4,4 & 4,7 & 6,0 & 6,0 \\
\hline
\end{tabular}

(1) $450 \mathrm{~kg}$ de peso vivo.

O efeito das camadas de solo nos modelos matemáticos não lineares das curvas de retenção de água e de resistência do solo à penetração foi testado estatisticamente pela variável indicadora com valores “-1", para a camada de 0-0,10 m; “0”, para a camada de 0,10-0,20 m; e "1", para a camada de 0,20-0,30 m (Neter et al., 1996; Tormena \& Silva, 2002; Fidalski et al., 2010). Os coeficientes de regressão dos modelos matemáticos das curvas de retenção de água e de resistência do solo à penetração foram estimados pelo método de Gauss-Newton, com o procedimento PROC NLIN (SAS, 1999), sendo selecionados os coeficientes das curvas de retenção de água do solo pelos intervalos de confiança sem valor nulo e os coeficientes das curvas de resistência do solo à penetração pela probabilidade $(\mathrm{p}<0,05)$.

O IHO foi calculado para cada um dos valores de Ds, utilizando-se as curvas de retenção de água do solo e as curvas de resistência do solo à penetração, considerando-se os limites críticos de -80 hPa, - $15.000 \mathrm{hPa}, 2,5 \mathrm{MPa}$ e $0,10 \mathrm{~m}^{3} \mathrm{~m}^{-3}$, respectivamente, para $\theta_{\mathrm{cc}}, \theta_{\mathrm{pmp}}, \theta_{\mathrm{rp}}$ e $\theta_{\mathrm{pa}}$ (Tormena et al., 1998, 1999). Após conseguir o IHO, foram obtidas as Dsc, tomada como o valor de Ds, em que o $\mathrm{IHO}=0$ (Tormena et al., 1998, 1999), para cada uma das três camadas de solo. A partir dos 90 valores de Ds, obtevese a frequência relativa de amostras de solo com maior restrição física ( $\mathrm{Ds} \geq \mathrm{Dsc}$ ), tabuladas para tratamento e camada de solo.

Os dados de Ds foram submetidos à análise de variância pelo modelo matemático de blocos ao acaso, no esquema de parcelas subsubdivididas (Banzatto \& Kronka, 2006). As parcelas (três camadas), as subparcelas (cinco tratamentos de alturas de pastejo), as subsubparcelas (duas amostragem de solo duplicata) e a comparação de médias (Teste F; p<0,05) foram feitas pelo teste de Tukey $(\mathrm{p}<0,05)$.

\section{RESULTADOS E DISCUSSÃO}

Os tratamentos de altura de pastejo $(0,10 ; 0,20$; 0,30 ; e $0,40 \mathrm{~m}$ e sem pastejo) não alteraram a Ds $(\mathrm{p}<0,05)$, após três safras agrícolas de lavoura (aveia/ soja) e o primeiro ano de integração lavoura-pecuária (soja e B. ruziziensis) (Figura 1). Outros autores

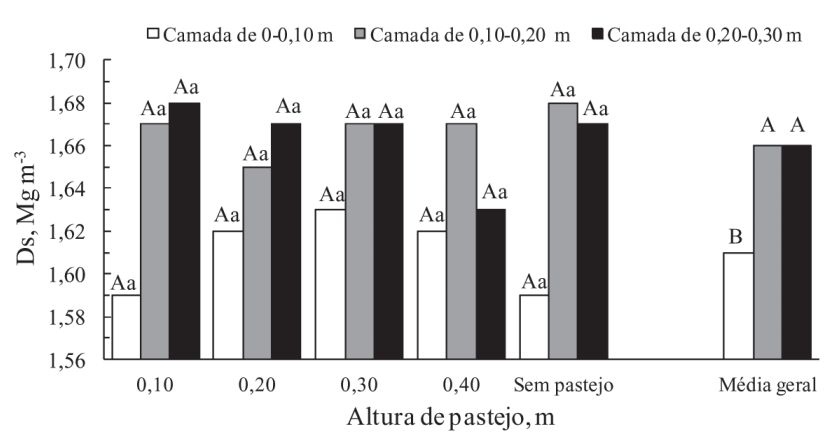

Figura 1. Densidade do solo (Ds) para os tratamentos de altura de pastejo $(0,10 ; 0,20 ; 0,30 ;$ e $0,40 \mathrm{~m}$ e sem pastejo) e média dos cinco tratamentos nas camadas de $0-0,10 ; 0,10-0,20 ;$ e $0,20-0,30 \mathrm{~m}$ do Latossolo Vermelho distrófico típico, em sistema de integração lavoura-pecuária. Letras maiúsculas comparam as camadas e letras minúsculas, os tratamentos de altura de pastejo e sem pastejo. Médias seguidas pela mesma letra não diferem entre si pelo teste Tukey $(p<0,05)$.

também não verificaram a influência de tratamentos em sistemas integração lavoura-pecuária (Marchão et al., 2007; Spera et al., 2010; Conte et al., 2011; Moreira et al., 2012).

A Ds na camada de $0-0,10 \mathrm{~m}\left(1,61 \mathrm{Mg} \mathrm{m}^{-3}\right)$ foi menor do que nas camadas de 0,10-0,20 e 0,20-0,30 m $\left(1,66 \mathrm{Mg} \mathrm{m}^{-3}\right)$ (Figura 1). Esses valores de Ds também foram registrados em solo da mesma classe pedológica no noroeste do Paraná na camada de 0-0,75 m, após cinco anos de pastejo contínuo de capim coast cross (C. dactylon), com taxas de lotações de 3,75 a 4,75 UA ha-1 (Fidalski et al., 2008a).

O tráfego de trator e os equipamentos utilizados para o manejo das culturas e da pastagem não promoveram aumentos de Ds (Figura 1), comparandose aos valores de Ds desses solos com aqueles de solo sob braquiárias, sob tráfego contínuo de trator e equipamentos em pomares de laranja, após 10 a 18 anos em solo similar (Fidalski et al., 2012). Utilizandose as Ds máximas para os Latossolos Vermelhos distróficos típicos, 1,87 e $1,80 \mathrm{Mg} \mathrm{m}^{-3}$, respectivamente para as camadas de 0-0,10 e 0,10-0,20 m (Fidalski et al., 2012), ter-se-iam graus de compactação das camadas de 0-0,10 e 0,10-0,20 m, respectivamente, 
de 86 e $92 \%$, similares aos valores obtidos por Fidalski et al. (2012). Esses resultados permitem caracterizar a maior susceptibilidade à compactação do solo na camada de 0,10-0,20 m, em razão das características desses solos com transição arenosa e média entre os horizontes A e Bw (Quadro 1).

Os coeficientes das curvas de retenção de água $[\theta(\psi)]$ do solo ajustados ao modelo de van Genuchten explicaram $99 \%(\mathrm{p}<0,0001)$ da variabilidade do $\theta$. Não foi constatado efeito dos tratamentos nos coeficientes, apenas das camadas e da Ds, respectivamente para os coeficientes $\theta \mathrm{r}$ e $n$ (quadro $3)$. Os valores dos coeficientes $(\alpha, \theta$ s, $\theta$ r e $n$ ) estão dentro da amplitude dos valores obtidos em solos similares por Fidalski \& Tormena (2007) e Machado et al. (2008), confirmando a viabilidade técnica de se obterem curvas de retenção de água do solo (Quadro 3 ) por meio de tensiômetro em miniatura (Eijkelkamp, 2012) e psicrômetro (Klein et al., 2010), considerando-se as vantagens de maior rapidez associadas aos menores custos desses equipamentos comparados às câmaras de Richards.

Os coeficientes das curvas de resistência do solo à penetração do modelo proposto por Busscher (1990) explicaram $84 \%(\mathrm{p}<0,0001)$ da variabilidade da RP em razão de $\theta$ e Ds. Não houve efeito dos tratamentos nesses coeficientes, apenas das camadas de solo, que influenciaram os coeficientes do intercepto e o coeficiente de $\theta$, resultando em curvas de resistência do solo à penetração para cada uma das três camadas (Quadro 3). O coeficiente de $\theta$ indica que a RP aumenta com mais intensidade em profundidade para o mesmo valor de $\theta$, corroborando os resultados de Costa et al. (2012). Cavalieri et al. (2006) obteve valor de -2,154 para esse coeficiente, independentemente das camadas de $0-0,15$ e $0-0,30 \mathrm{~m}$ em solo de mesma classe pedológica e textural, próximos ao valor obtido por Araújo et al. (2004), também em solo similar. O modelo ajustado explicou $84 \%$ da variabilidade dos dados de RP, similares aos ajustes obtidos por Araújo et al. (2004) e Cavalieri et al. (2006), os quais utilizaram penetrômetro de bancada descrito por Tormena et al. (1998). As curvas de resistência do solo à penetração obtidas com o minipenetrômetro dinâmico foram tecnicamente viáveis e conseguidas com equipamento de menor custo (Sá et al., 2007).

O IHO foi maior na camada de $0-0,10 \mathrm{~m}$, decrescendo nas camadas de 0,10-0,20 e 0,20-0,30 m (Figura 2), em razão do aumento da $\mathrm{RP}$ que foi o fator mais limitante do IHO para todos os valores de Ds (Figura 2a,b,c), corroborando aos resultados obtidos em solos similares (Araújo et al., 2004; Fidalski et al., 2010) e em integração lavoura-pecuária (Serafim et al., 2008; Petean et al., 2010). Não houve restrição do IHO pela $\theta_{\mathrm{pa}}$, com valores desse indicador superiores ao limite crítico de $0,10 \mathrm{~m}^{3} \mathrm{~m}^{-3}$ (Figura 2), que são característicos de classes texturais arenosa e média (Quadro 1), constatadas também por Fidalski \& Tormena (2007) e Fidalski et al. (2010).

O maior valor de IHO na camada superficial arenosa está associado ao maior teor de carbono orgânico (Quadro 1). Fidalski et al. (2008b) incorporaram o carbono orgânico nos ajustes das curvas de retenção de água do solo e curvas de resistência do solo à penetração, que verificaram maior $\theta$ e menor RP, refletindo em maior IHO no tratamento com gramínea (Fidalski et al., 2010). O uso e manejo desses solos com gramínea atendem a aptidão agrícola desses solos (Embrapa, 1984), que estão recobertos por pastagem em $70 \%$ da área agrícola do noroeste do Paraná. Nesse contexto, o manejo do solo no sistema integração lavoura-pecuária com a manutenção de disponibilidade de forrageiras constitui opção viável para a manutenção da sua qualidade física.

Os valores da Dsc foram de 1,66; 1,64; e 1,62 $\mathrm{Mg} \mathrm{m}^{-3}$ respectivamente para as camadas de $0-0,10 ; 0,10-0,20$; e $0,20-0,30 \mathrm{~m}$ (Figura 3 ) e caracterizam maior capacidade de carga de máquinas e pisoteio dos animais no aumento da compactação na camada superficial $(0-0,10 \mathrm{~m})$ do que nas subsuperficiais $(0,10$ 0,20 e 0,20-0,30 m). Em Latossolo Vermelho distrófico textura média, Cavalieri et al. (2006) obtiveram Dsc de 1,65 e $1,62 \mathrm{Mg} \mathrm{m}^{-3}$, respectivamente para as camadas de $0-0,15$ e $0,15-0,30 \mathrm{~m}$. Contrariamente, Petean et al. (2010) verificaram o aumento da Dsc da camada de 0-0,075 para 0,075-0,150 m em um

Quadro 3. Curvas de retenção de água e curvas de resistência do solo à penetração do Latossolo Vermelho distrófico típico em três camadas em sistema de integração lavoura-pecuária

\begin{tabular}{|c|c|}
\hline Camada & Curva \\
\hline \multicolumn{2}{|l|}{$\mathrm{m}$} \\
\hline & Curva de retenção de água do solo \\
\hline $0-0,10$ & $\left.\theta=0,0689+\left\{(\theta \mathrm{s}-0,0689) /\left[1+(0,0321 \psi)^{[3,2266-(0,9758 \mathrm{xDs})}\right]\right]^{(1-1 /[3,2266-(0,9758 \mathrm{xDs})]}\right\}$ \\
\hline $0,10-0,20$ & $\theta=0,0729+\left\{(\theta \mathrm{s}-0,0729) /\left[1+(0,0321 \psi)^{[3,2266-(0,9758 \mathrm{xDs})]}\right]^{(1-1 /[3,2266-(0,9758 \mathrm{xDs})]}\right\}$ \\
\hline $0,20-0,30$ & $\theta=0,0769+\left\{(\theta \mathrm{s}-0,0769) /\left[1+(0,0321 \psi)^{[3,2266-(0,9758 \mathrm{xDs})]}\right]^{(1-1 /[3,2266-(0,9758 \mathrm{xDs})]}\right\}$ \\
\hline & Curva de resistência do solo à penetração \\
\hline $0-0,10$ & $\mathrm{RP}=0,0123 \theta^{-1,2762} \mathrm{Ds}^{5,6262}$ \\
\hline $0,10-0,20$ & $\mathrm{RP}=0,0049 \theta^{-1,8467} \mathrm{Ds}^{5,6262}$ \\
\hline $0,20-0,30$ & $\mathrm{RP}=0,0019 \theta^{-2,4172} \mathrm{Ds}^{5,6262}$ \\
\hline
\end{tabular}


Latossolo Vermelho distroférrico muito argiloso, sob integração lavoura-pecuária. De modo similar, Calonego et al. (2011) constataram aumento da Dsc em Nitossolo Vermelho sob B. brizantha, na camada de $0,20-0,40 \mathrm{~m}$.

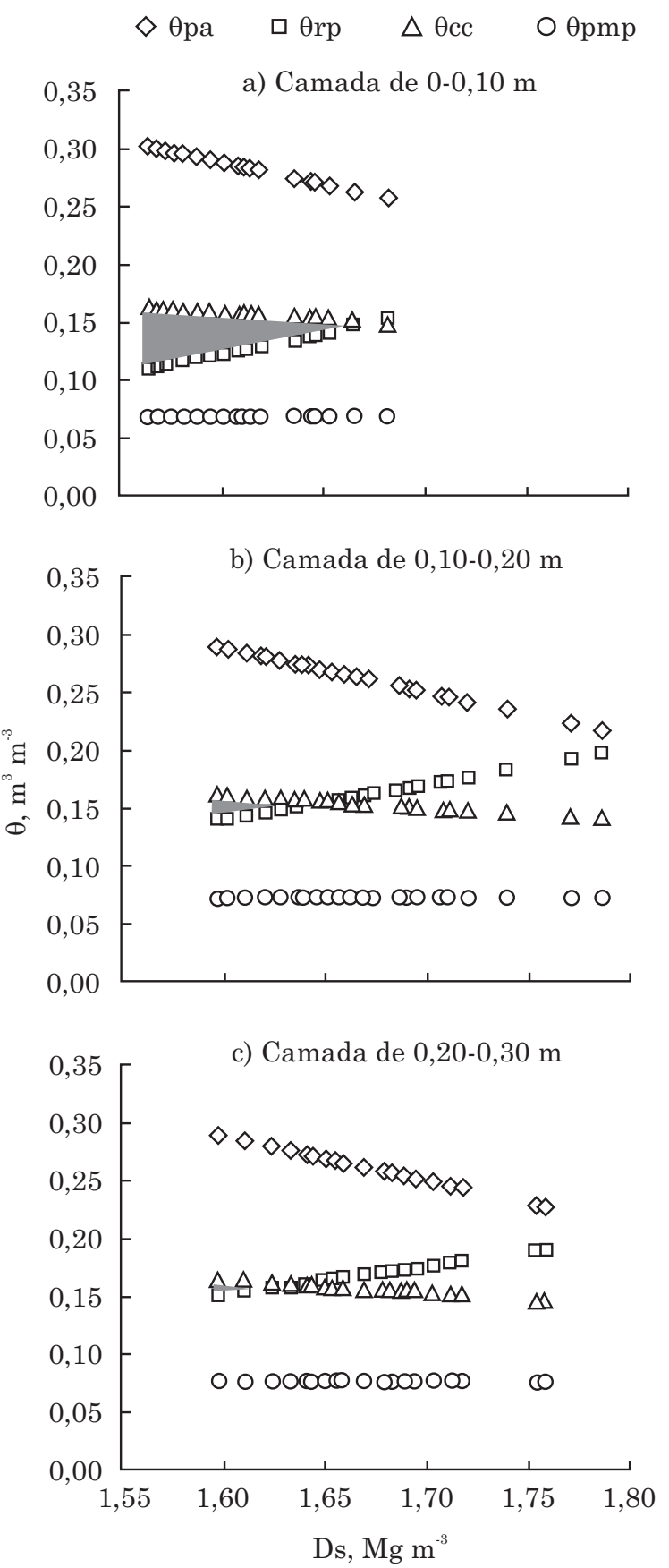

Figura 2. $\theta$ em função da Ds para as camadas de 00,10 (a), 0,10-0,20 (b) e 0,20-0,30 m (c) do Latossolo Vermelho distrófico típico em sistema de integração lavoura-pecuária. As áreas hachuradas em cinza correspondem ao IHO, para os limites críticos de -80 hPa, $-15.000 \mathrm{hPa}$, 2,5 MPa e $0,10 \mathrm{~m}^{3} \mathrm{~m}^{-3}$, respectivamente para $\theta_{\mathrm{cc}}$, $\theta_{\mathrm{pmp}}, \theta_{\mathrm{rp}} \mathrm{e} \theta_{\mathrm{pa}}$.
Os resultados deste trabalho corroboram as recomendações de Karlen et al. (1997) e Sojka \& Upchurch (1999), ao definirem indicadores de qualidade de solo específico para determinada classe desse com uso e manejo. Portanto, o controle da qualidade física de Latossolo Vermelho distrófico, típico do noroeste do Paraná, sob o sistema de integração lavoura-pecuária com $B$. ruziziensis e soja deverá manter valores de $\mathrm{Ds}$ inferiores às Dsc de referências 1,$66 ; 1,64 ;$ e $1,62 \mathrm{Mg} \mathrm{m}^{-3}$, respectivamente para as camadas de $0-0,10 ; 0,10-0,20$; e $0,20-0,30 \mathrm{~m}$, mantendo-se o manejo com altura mínima de disponibilidade de forrageira de $0,10 \mathrm{~m}$ para o pastejo; administrar adequadamente o uso de máquinas e equipamentos em condições de umidade do solo elevadas; e não exceder o limite de máquinas que vem sendo utilizadas, que poderiam propiciar a degradação física desse solo pelo aumento da sua Ds.

Neste trabalho, foram constatadas outras contribuições técnicas: proporcionar viabilidade de uso equipamentos de menor custo e de maior rapidez para descrever a curva de retenção de água do solo (tensiômetro em miniatura e psicrômetro) e a curva de resistência do solo à penetração (minipenetrômetro dinâmico) (Sá et al., 2007); e identificar a camada de 0,10-0,20 m como a de maior limitação física e hídrica desse Latossolo Vermelho distrófico típico, em razão de estar localizada entre os horizontes A e Bw (Quadro 1). Essa camada é constituída por solo arenoso e com teores intermediários de carbono orgânico, que com o aumento da sua Ds eleva a $\mathrm{RP}$, reduzindo abruptadamente o IHO (Figuras 2 e 3). Para reduzir os efeitos da RP nesses solos, deve-se priorizar o manejo com gramíneas para manter os teores de

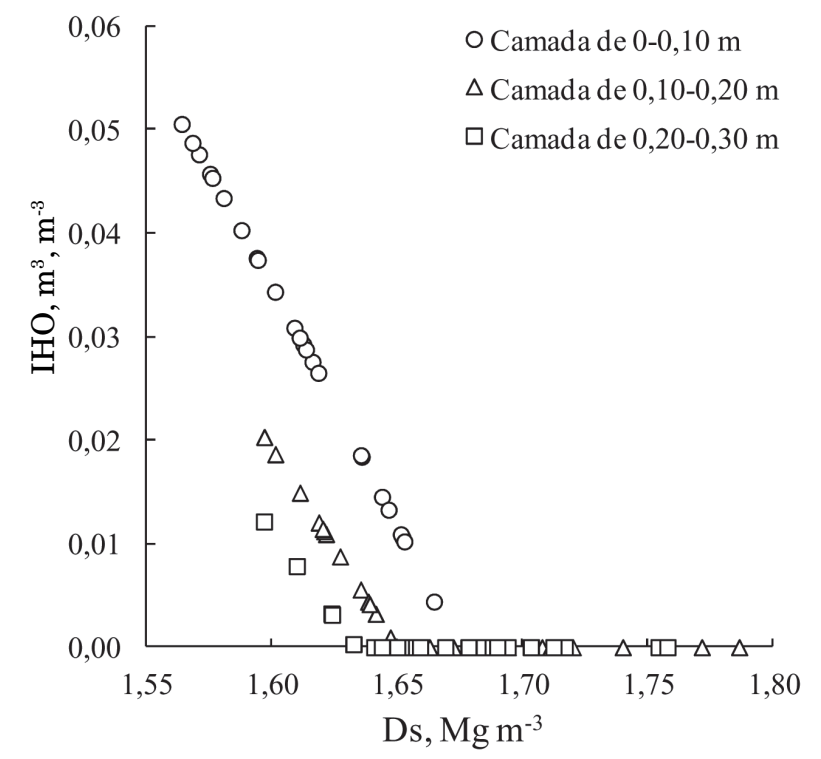

Figura 3. Intervalo hídrico ótimo (IHO) em função da Ds para as camadas de $0-0,10 ; 0,10-0,20$; e 0,20-0,30 m do Latossolo Vermelho distrófico típico, em sistema de integração lavourapecuária. 
carbono orgânico, o maior IHO e a disponibilidade de água para as plantas (Fidalski \& Tormena, 2007; Fidalski et al., 2008b; Silva et al., 2009; Fidalski et al., 2010a).

A frequência relativa de amostras de solo com Ds $\geq$ Dsc foi influenciada pelo tratamento de altura de pastejo e pela camada de solo (Figura 4). O tratamento com altura de pastejo de $0,30 \mathrm{~m}$ apresentou a maior restrição na camada de $0-0,10 \mathrm{~m}$ ( $50 \%$ ), seguido do de $0,10 \mathrm{~m}$, na camada de $0,10-0,20$ $\mathrm{m}(100 \%)$; todos os tratamentos apresentaram elevada restrição na camada de 0,20-0,30 m (83-100 \%). Considerando-se os tratamentos independentemente das três camadas de solo, as frequências relativas por tratamento foram de $67,50,61,50$ e $61 \%$, respectivamente para os tratamentos de altura de pastejo 0,10; 0,20; 0,30; e 0,40 m e sem pastejo. Esses resultados caracterizaram que o tratamento de altura de pastejo de $0,10 \mathrm{~m}$ foi mais restritivo após o primeiro período de pastejo nesse sistema de integração lavourapecuária, em razão da maior lotação de bovinos (Quadro 2). Petean et al. (2010) também verificaram menor qualidade física com a menor altura de pastejo de aveia e azevém pelo aumento de Ds superiores a Dsc na camada de 0-0,075 m de um Latossolo Vermelho distroférrico, sob plantio direto em sistema de integração lavoura-pecuária. Neste estudo, a maior degradação da qualidade física do solo ocorreu na camada de 0,10-0,20 m, que corresponde à camada de solo do arenito no noroeste do Paraná, que apresentou maior grau de compactação (Fidalski et al., 2012).

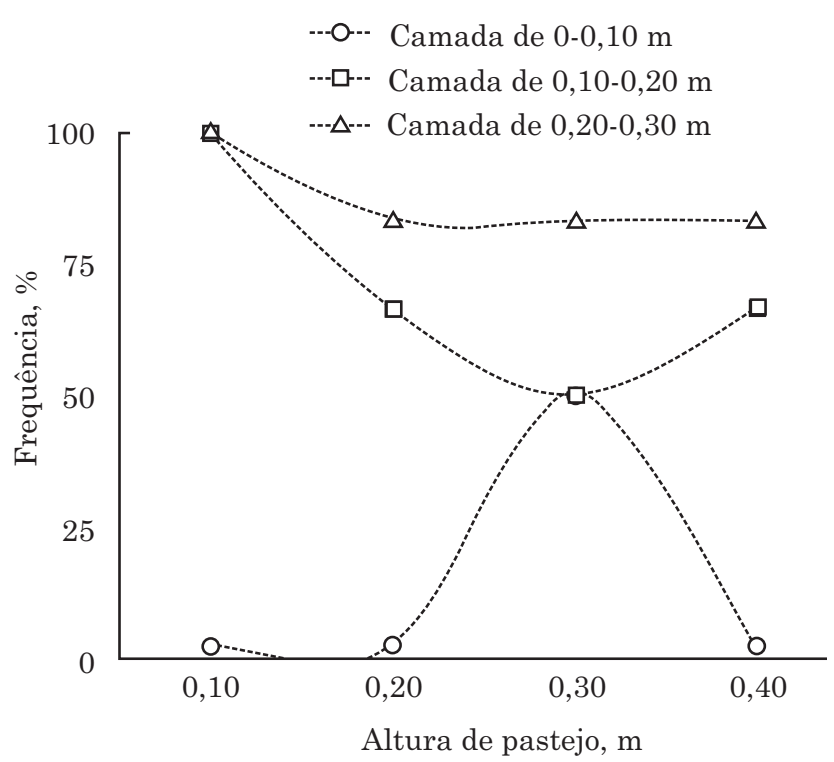

Figura 4. Frequência relativa de amostras de solo com Dse $\geq$ Dsc para os tratamentos de altura de pastejo $(0,10 ; 0,20 ; 0,30$; e $0,40 \mathrm{~m}$ e sem pastejo) nas camadas de $0-0,10 ; 0,10-0,20 ;$ e $0,20-0,30 \mathrm{~m}$ do Latossolo Vermelho distrófico típico, em sistema de integração lavoura-pecuária.

\section{CONCLUSÕES}

1. O IHO foi maior a $0-0,10 \mathrm{~m}$ e a RP foi o fator de maior limitação do IHO em todas as camadas, especialmente nas 0,10-0,20 e 0,20-0,30 m.

2. As Dsc decresceram em profundidade de 1,66; 1,$64 ; \mathrm{e} 1,62 \mathrm{Mg} \mathrm{m}^{-3}$, respectivamente para as camadas de $0-0,10 ; 0,10-0,20$ e $0,20-0,30 \mathrm{~m}$.

3. O manejo desse solo sob sistema integração lavoura-pecuária com altura de pastejo de $0,10 \mathrm{~m}$ apresentou a maior frequência de amostras de solo com Ds $\geq$ Dsc.

\section{LITERATURA CITADA}

ARAÚJO, M.A.; TORMENA, C.A. \& SILVA, A.P. Propriedades físicas de um Latossolo Vermelho distrófico cultivado e sob mata nativa. R. Bras. Ci. Solo, 28:337-345, 2004.

AGUINAGA, A.A.Q.; CARVALHO, P.C.F.; ANGHINONI,I.; SANTOS, D.T.; FREITAS, F.K. \& LOPES, M.T. Produção de novilhos superprecoces em pastagem de aveia e azevém submetida a diferentes alturas de manejo. R. Bras. Zootec., 35:1765-1773, 2006.

BANZATTO, D.A. \& KRONKA, S.N. Experimentação agrícola. 4.ed. Jaboticabal, Funep, 2006. 237p.

BUSSCHER, W.J. Adjustment of flat-tipped penetrometer resistance data to a common water content. Trans. Am. Soc. Agric. Eng., 33:519-524, 1990.

CALONEGO, J.C.; BORGHI, E. \& CRUSCIOL C.A.C. Intervalo hídrico ótimo e compactação do solo com cultivo consorciado de milho e braquiária. R. Bras. Ci. Solo, 35:2183-2190, 2011

CAVALIERI, K.M.V.; TORMENA, C.A.; VIDIGAL FILHO, P.S.; GONÇALVES, A.C.A. \& COSTA, A.C.S. Efeitos de sistemas de preparo nas propriedades físicas de um Latossolo Vermelho distrófico. R. Bras. Ci. Solo, 30:137147, 2006.

CAVIGLIONE, J.H.; KIIHL, L.R.B.; CARAMORI, P.H. \& OLIVEIRA, D. Cartas climáticas do Paraná. Londrina, IAPAR, 2000. CD ROM

CLAESSEN, M.E.E., org. Manual de métodos de análise de solo. 2.ed. Rio de Janeiro, Embrapa-CNPS, 1997. 212p. (Documentos, 1)

COLLARES, G.L.; REINERT, D.J.; REICHERT, J.M. \& KAISER, D.R. Compactação superficial de Latossolos sob integração lavoura-pecuária de leite no noroeste do Rio Grande do Sul. Ci. Rural, 41:246-250, 2011.

CONTE, O.; FLORES, J.P.C.; CASSOL, L.C.; ANGHINONI, I.; CARVAlHO, P.C.F.; LEVIEN, R. \& WESP, C.L. Evolução de atributos físicos de solo em sistema de integração lavoura-pecuária. Pesq. Agropec. Bras., 46:1301-1309, 2011. 
COSTA, M.A.T.; TORMENA, C.A.; LUGÃO, S.M.B.; FIDALSKI, J.; NASCIMENTO, W.G. \& MEDEIROS, F.M. Resistência do solo à penetração e produção de raízes e de forragem em diferentes níveis de intensificação do pastejo. R. Bras. Ci. Solo, 36:993-1004, 2012.

EIJKELKAMP. T5 Miniature Pressure Transducer Tensiometer. Disponível em: <http://pkd. eijkelkamp.com/Portals/2/Eijkelkamp/Files/Manuals/ M1-14041002e\%20Tensior\%20T5.pdf> Acesso em: 21 jun. 2012.

EMPRESA BRASILEIRA DE PESQUISA AGROPECUÁRIA - EMBRAPA. Levantamento de reconhecimento dos solos do Estado do Paraná. Londrina, Embrapa-SNLCS/ Sudesul/Iapar, 1984. v.1/2. (Embrapa-SNLCS. Boletim de Pesquisa, 27; IAPAR. Boletim Técnico, 16)

FASOLO, P.J.; CARDOSO, A.P.; HOCHMÜLLER, D.P.; RAUEN, M.J. \& PÖTTER, R.O. Erosão: Inventário de áreas críticas no Noroeste do Paraná. Londrina, IAPAR, 1988. 20p. (Boletim Técnico, 23)

FIDALSKI, J. \& TORMENA, C.A. Funções de pedotransferência para as curvas de retenção de água e de resistência do solo à penetração em sistemas de manejo com plantas de cobertura permanente em citros. Ci. Rural, 37:1316-1322, 2007.

FIDALSKI, J.; TORMENA, C.A.; CECATO, U.; BARBERO, L.M.; LUGÃO, S.M.B. \& COSTA, M.A.T. Qualidade física do solo em pastagem adubada e sob pastejo contínuo. Pesq. Agropec. Bras., 43:1583-1590, 2008a.

FIDALSKI, J.; MARUR, C.J. \& TORMENA, C.A. Respostas fisiológicas da laranjeira 'Pêra' aos sistemas de manejo de cobertura permanente do solo nas entrelinhas. R. Bras. Ci. Solo, 32:1307-1317, 2008b.

FIDALSKI, J.; TORMENA, C.A. \& SILVA, Á.P. Least limiting water range and physical quality of soil under groundcover management systems in citrus. Sci. Agric., 67:448-453, 2010.

FIDALSKI, J.; TORMENA, C.A.; AULER, P.A.M. \& ANGHINONI, G. Grau de compactação de Argissolo e Latossolo em pomares de laranja implantados com preparo convencional e em faixas. In: REUNIÃO BRASILEIRA DE MANEJO E CONSERVAÇÃO DO SOLO E DA ÁGUA, 19., Lages, 2012. Anais... Viçosa, MG, Sociedade Brasileira de Ciência do Solo, 2012.

KARLEN, D.L.; MAUSBACK, M.J.; DORAN, J.W.; CLINE, R.G.; HARRIS, R.F. \& SCHUMAN, G.E. Soil quality: A concept, definition, and framework for evolution. Soil Sci. Soc. Am. J., 61:4-10, 1997.

KLEIN, V.A.; BASEGGIO, M.; MADALOSSO, T. \& MARCOLIN, C.D. Testura do solo e a estimativa do teor de água no ponto de murcha permanente com psicrômetro. Ci. Rural, 40:1550-1556, 2010.

MACHADO, J.L.; TORMENA, C.A.; FIDALSKI, J. \& SCAPIM, C.A. Inter-relações entre as propriedades físicas e os coeficientes da curva de retenção de água de um Latossolo sob diferentes sistemas de uso. R. Bras. Ci. Solo, 32:495-502, 2008.
MARCHÃO, R.L.; BALBINO, L.C.; SILVA, E.M.; SANTOS JUNIOR, J.D.G.; SÁ, M.A.C.; VILELA, L. \& BECQUER, T. Qualidade física de um Latossolo Vermelho sob sistemas de integração lavoura-pecuária no Cerrado. Pesq. Agropec. Bras., 42:873-882, 2007.

MOREIRA, W.H.; BETIOLI JUNIOR, E.; PETEAN, L.P.; TORMENA, C.A.; ALVES, S.J.; COSTA. M.A.T. \& FRANCO, H.H.S. Atributos físicos de um Latossolo Vermelho distroférrico em sistema de integração lavourapecuária, R. Bras. Ci. Solo, 36:389-400, 2012.

MOTT, G.O. \& LUCAS, H.L. The design, conduct and interpretation of grazing trials on cultivated and improved pastures. In: INTERNATIONAL GRASSLAND CONGRESS, 6., 1952, Pennsylvania. Proceedings... Pennsylvania, State College Press, 1952. p.1380-1385.

NETER, J.; KUTNER, M.H.; NACHTSHEIM, C.J. \& WASSERMAN, W., eds. Applied linear statistical models. 4.ed. Chicago, IRWIN, 1996. 1408p.

PETEAN, L.P.; TORMENA, C.A. \& ALVES, S.J. Intervalo hídrico ótimo de um Latossolo Vermelho distroférrico sob plantio direto em sistema de integração lavoura-pecuária. R. Bras. Ci. Solo, 34:1515-1526, 2010.

SÁ, J.P.G. \& CAVIGLIONE, J.H. Arenito Caiuá: Capacidade de lotação das pastagens. Londrina, IAPAR, 1999. 15p. (Informe de Pesquisa, 132)

SÁ, M.A.C.; SANTOS JUNIOR, J.D.G.; RESCK, D.V.S.; FERREIRA, E.A.B. \& FRANZ, C.A.B. Minipenetrômetro dinâmico para determinação da resistência à penetração em amostras de solo indeformadas. Pesq. Agropec. Bras., 42:1659-1662, 2007.

SANTOS, H.G.; JACOMINE, P.K.T.; ANJOS, L.H.C.; OLIVEIRA, V.A.; OLIVEIRA, J.B.; COELHO, M.R.; LUMBRERAS, J.F. \& CUNHA, T.J.F., ed. Sistema brasileiro de classificação de solos. 2.ed. Rio de Janeiro, Embrapa Solos, 2006. 306p.

SARMENTO, P.; RODRIGUES, L.R.A.; CRUZ, M.C.P.; LUGÃO, S.M.B.; CAMPOS, F.P.; CENTURION, J.F. \& FERREIRA, M.E. Atributos químicos e físicos de um Argissolo cultivado com Panicum maximum Jacq. cv. IPR 86 Milênio, sob lotação rotacionada e adubado com nitrogênio. R. Bras. Ci. Solo, 32:183-193, 2008.

SERAFIM, M.E.; VITORINO, A.C.T.; PEIXOTO, P.P.P.; SOUZA, C.M.A. \& CARVALHO, D.F. Intervalo hídrico ótimo em um Latossolo Vermelho distroférrico sob diferentes sistemas de produção. Eng. Agríc., 28:654-665, 2008.

SILVA, A.P.; KAY, B.D. \& PERFECT, E. Characterization of the least limiting water range. Soil Sci. Soc. Am. J., 58:1775-1781, 1994.

SILVA, Á.P.; TORMENA, C.A.; IMHOFF, S.D.C.; FIDALSKI, J. \& NEVES JUNIOR, A.F. Intervalo Hídrico Ótimo e sua importância para as plantas. Tópicos em ciência do solo. Viçosa, MG, Sociedade Brasileira de Ciência do Solo, 2009. v.6. p.1-30.

SPERA, S.T.; SANTOS, H.P.; FONTANELI, R.S. \& TOMM, G.O. Atributos físicos de um Hapludox em função de sistemas de produção integração lavoura-pecuária (ILP), sob plantio direto. Acta Sci. Agron., 32:37-44, 2010. 
STATISTICAL ANALYSIS SYSTEM INSTITUTE - SAS. SAS/ STAT Procedure guide for personal computers. Version 5. Cary, 1999.

SOJKA, R.E. \& UPCHURCH, D.R. Reservations regarding the soil quality concept. Soil. Sci. Am. J., 63:1039-1054, 1999.

TORMENA, C.A. \& SILVA, A.P. Incorporação da densidade no ajuste de dois modelos à curva de retenção de água no solo. R. Bras. Ci. Solo, 26:305-314, 2002.

TORMENA, C.A.; SILVA, A.P. \& LIBARDI, P.L. Caracterização do intervalo hídrico ótimo de um Latossolo Roxo sob plantio direto. R. Bras. Ci. Solo, 22:573-581, 1998.
TORMENA, C.A.; SILVA, A.P. \& LIBARDI, P.L. Soil physical quality of a Brazilian Oxisol under two tillage systems using the least limiting water range approach. Soil Till. Res., 52:223-232, 1999.

TORMENA, C.A.; ARAÚJO, M.A.; FIDALSKI, J. \& COSTA, J.M. Variação temporal do intervalo hídrico ótimo de um Latossolo Vermelho distroférrico em sistemas de plantio direto. R. Bras. Ci. Solo, 31:211-219, 2007.

van GENUCHTEN, M.T. A closed-form equation for predicting the hydraulic conductivity of unsaturated soils. Soil Sci. Soc. Am. J., 44:892-898, 1980. 